\title{
Complete atrioventricular block during exercise: New insights from an old test
}

\author{
Eduardo M. Vilela, Susana Torres, Helena Gonçalves, João Primo, Madalena Teixeira, Pedro Braga \\ Cardiology Department, Vila Nova de Gaia/Espinho Hospital Centre, Vila Nova de Gaia, Portugal
}

\begin{abstract}
Exercise stress testing can have a central role in the assessment of cardiovascular disease. Contemporary data, however, has highlighted the added value of imaging modalities over the exercise electrocardiogram in the investigation of coronary artery disease. Given the physiological changes associated with exercise and the possibility to address other parameters such as rhythm changes and the chronotropic response, exercise stress testing with continuous electrocardiographic monitoring can still have an important place in contemporary clinical practice. We report the case of a complete atrioventricular block associated with exercise and discuss the current role of exercise stress testing with continuous electrocardiographic monitoring in this entity.
\end{abstract}

\section{Introduction}

Exercise testing can have an important role in the investigation of cardiovascular (CV) disease, in several clinical scenarios $[1,2]$. Although exercise stress testing with continuous electrocardiographic monitoring (ExECG) has being extensively used for the assessment of individuals with suspected coronary artery disease (CAD) [3], contemporary evidence has increasingly suggested the added value of imaging modalities [4]. This notion stems

Correspondence: Eduardo M. Vilela, Cardiology Department, Vila Nova de Gaia / Espinho Hospital Centre, Rua Conceição Fernandes, 4434-502, Vila Nova de Gaia, Portugal.

E-mail: eduardomvilela@gmail.com

Key words: Complete heart block; exercise stress test; syncope.

Contributions: All authors contributed equally to the present article.

Conflict of interest: The authors have no conflict of interest regarding this article.

Received for publication: 12 October 2018.

Accepted for publication: 4 January 2019.

CC Copyright E.M. Vilela et al., 2019

Licensee PAGEPress, Italy

Monaldi Archives for Chest Disease 2019; 89:1002

doi: 10.4081/monaldi.2019.1002

This article is distributed under the terms of the Creative Commons Attribution Noncommercial License (by-nc 4.0) which permits any noncommercial use, distribution, and reproduction in any medium, provided the original author(s) and source are credited. greatly from the increased diagnostic performance of imaging stress tests when compared to ExECG [4,5]. Even though considering the hinderances associated with ExECG, this test can still yield important data beyond the classical assessment of the ST-T segment [6]. Specifically, the possibility of assessing cardiac rhythm changes as well as the chronotropic response to exercise can be of pivotal clinical importance [6-8].

\section{Case Report}

We present the case of an active 72-year-old man who was referred to an ExECG due to de novo recurring episodes of light headedness and fatigue during his daily activities. His prior medical history included hypertension, dyslipidemia and obesity. His current medication included an angiotensin-converting enzyme inhibitor, a thiazide diuretic, a statin and acetylsalicylic acid. The patient's physical examination was unremarkable, and no metabolic disturbances were present. His resting ECG showed sinus rhythm (SR) with a borderline PR interval as well as a left anterior hemiblock and a right bundle branch block (Figure 1). Prior to the ExECG he had undergone a transthoracic echocardiogram which revealed a mildly dilated left atrium, as well as Holter monitoring which showed SR with frequent premature supra-ventricular complexes as well as the presence of premature ventricular complexes (PVCs), with no other significant abnormalities.

His ExECG (standard Bruce protocol on a treadmill) was unremarkable except for the presence of occasional isolated PVCs up to about 07:30 minutes of the test when it was interrupted due to general discomfort and subsequent syncope with documentation of a complete atrioventricular block (CAVB), first with no ventricular escape rhythm and afterwards (at about 30 seconds of recovery) with an escape rhythm of circa 30 beats per minute (Figure 2A-C). The patient was managed in accordance with the European Resuscitation Council guidelines [9], and a temporary transvenous pacemaker was implanted given lack of recovery from the CAVB despite exercise interruption and interim management [9]. Given the patients' global CV risk profile and the presentation of CAVB during exercise, cardiac catheterization (CC) was performed as to exclude $\mathrm{CAD}$. His $\mathrm{CC}$ demonstrated right coronary dominance, and no significant epicardial CAD was present (in neither the left or right coronary arteries or main branches). Given the clinical scenario presented, a permanent pacemaker was implanted, with the patient being rendered asymptomatic.

\section{Discussion}

CAVB during exercise is an infrequent finding [8,10-13]. A contemporary systematic review of the literature proposes several 
potential mechanisms for this phenomenon [10]. Although intrinsic conduction tissue disease seems of paramount importance $[8,10]$, an ischemic contribution can also be present and should be excluded $[10,11,13]$. Indeed, an ischemic defect affecting the atrioventricular (AV) node has been described as a possible cause of exercise-induced CAVB $[10,13,14]$. Besides significant epicardial $\mathrm{CAD}$, arterial vasospasm has also been described a possible cause of CAVB [15]. In the present case, however, both etiologies seem unlikely, given the absence of significant epicardial CAD (specifically in the right coronary artery) and the absence of chest pain or ST segment elevation prior to the development of CAVB during the exercise test. Beyond an arterial component, venous obstruction could also lead to CAVB, possibly due to vagal imbalances as postulated by Simantirakis et al. [16]. Although in the present case a specific venous study was not done, the patients overall clinical history and the lack of abnormal findings on the physical examination make this hypothesis highly unlikely.

One limitation of the present report relates to the absence of an electrophysiological study (EPS). As previously described, most cases of reported exercise induced CAVB are infranodal $[10,11,17]$, though there are also reports describing a supranodal block [8], suggesting the utility of EPS to ascertain the specific site in question $[8,10,13]$. One possible explanation for exercise induced CAVB, as previously described $[10,11,13]$, derives from the differences in the conduction properties and refractory periods between the AV node (with increased conduction and decreased refractory period associated with exercise) and the His-Purkinje system (not under autonomic control, and as such whose refractory period is not substantially altered by exercise). Contrary to the physiologic response elicited in a normal conduction system, in the presence of conduction tissue disease (such as the case presented, given the baseline ECG characteristics) the diseased His-Purkinje system could thus be unable to conduct the impulses form the rapidly conducting AV node, and as such lead to a CAVB $[10,11,13]$.

Although this caveat should be kept under consideration, the patients' clinical history (with symptoms elicited by the exercise test) and the lack of recovery of appropriate SR despite exercise termination, in the absence of possible reversible secondary causes (such as negative chronotropic agents or an ischemic component amenable to revascularization), were an indication for a permanent pacemaker implantation, and as such an EPS would not be of clear benefit in this specific instance.

As reported in the present case, ExECG can be of great relevance in the clinical assessment of $\mathrm{CV}$ disease. This non-invasive and ubiquitous modality can provide valuable data, and its overall functional assessment (namely, in terms not only of the ST seg-

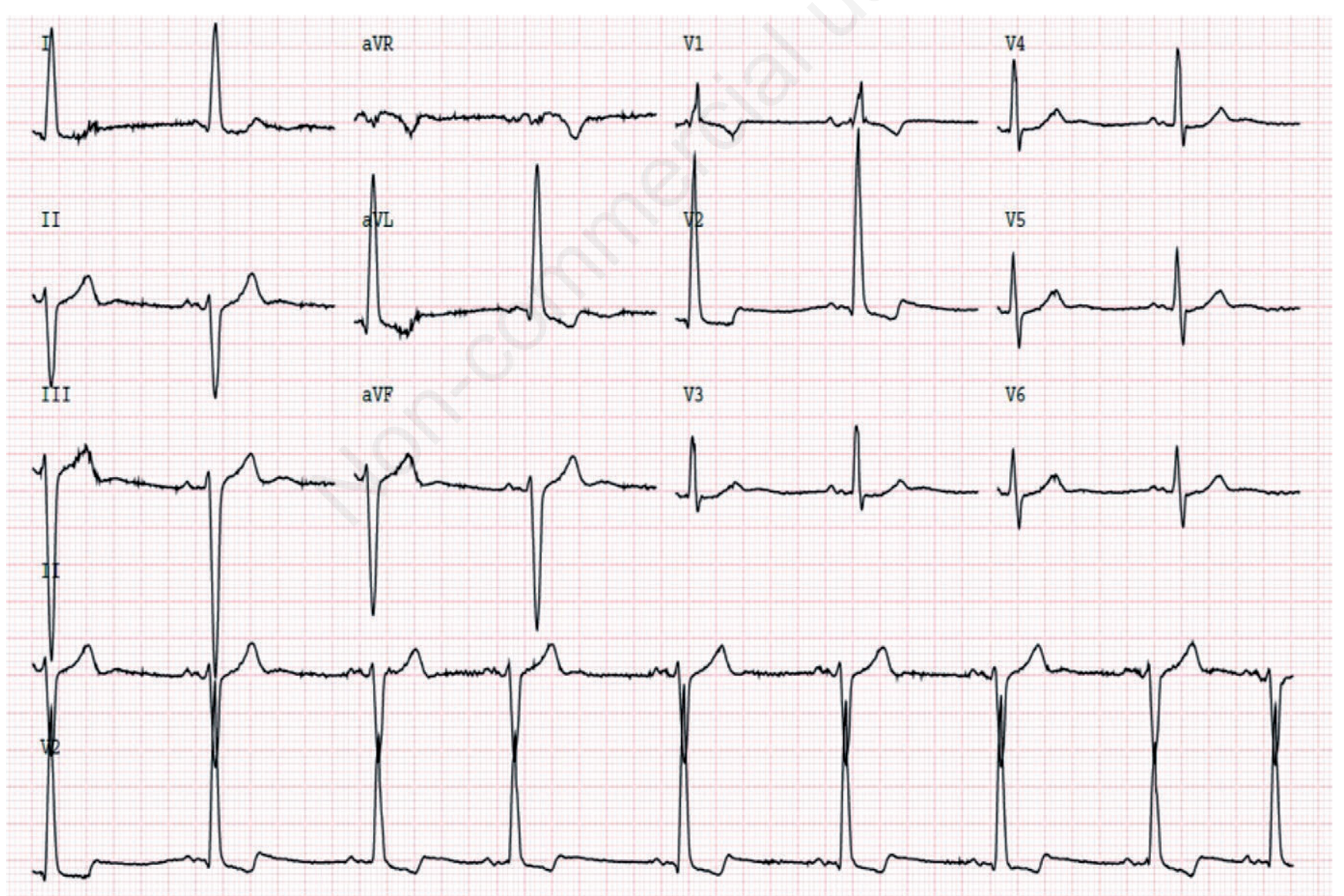

$25 \mathrm{~mm} / \mathrm{s} 10 \mathrm{~mm} / \mathrm{mV}$

Figure 1. Baseline electrocardiogram showing sinus rhythm with a borderline PR interval, left anterior hemiblock and right bundle branch block. 
ment but also of the rhythm and rate response) make it especially attractive in selected cases, such as the one presented. Albeit uncommon, the occurrence of a CAVB in the context of exercise should be considered, and although most patients present with changes in their baseline ECG some can have a normal resting ECG, thus highlighting the ability of ExECG testing to address rhythm changes during physiologic stress (as elicited by exercise) $[6,9,10]$. When taking into consideration the protean physiological changes during exercise [18-20], ExECG can be an interesting source of valuable clinical information.

\section{Conclusions}

This case highlights the importance of the ExECG in the investigation of a patient with atypical symptoms. Albeit the noted shortcomings associated with ExECG should be acknowledged in the era of imaging tests, this exam (and specifically its ability to assess functional status and address rhythm changes) can still play a central role in the holistic evaluation of several groups of patients, as illustrated in this report.

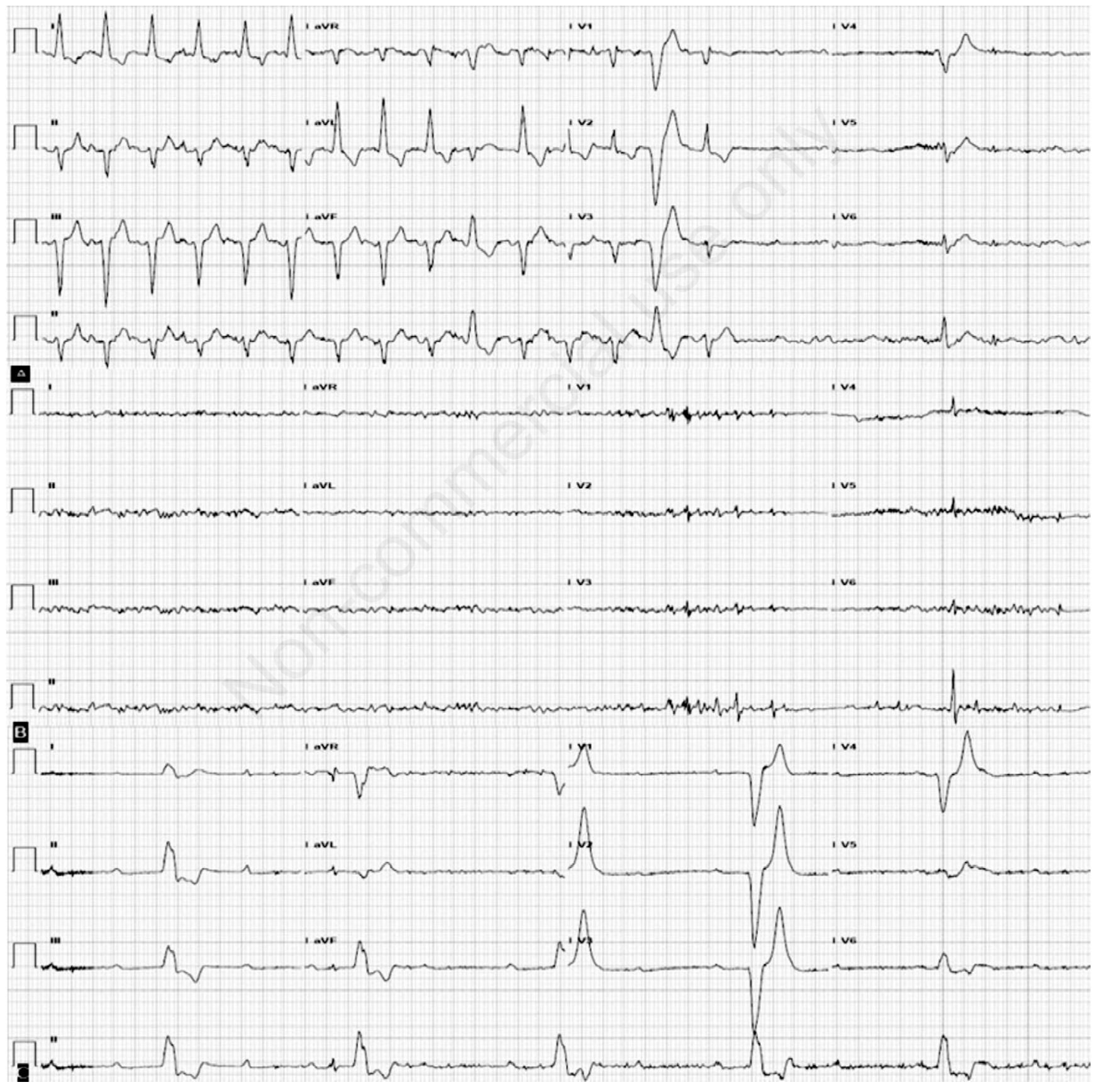

Figure 2. A-C) Onset of complete atrioventricular block during the exercise stress test. C) Complete atrioventricular block with an escape rhythm of about 30 beats per minute during the recovery phase of the exercise stress test. 


\section{References}

1. Guazzi M, Bandera F, Ozemek C, et al. Cardiopulmonary exercise testing: what is its value? J Am Coll Cardiol 2017;70: 1618-36

2. Gonzalez JA, Beller GA. Choosing exercise or pharmacologic stress imaging, or exercise ECG testing alone: How to decide. J Nucl Cardiol 2017;24:555-7.

3. Bourque JM, Beller GA. Value of exercise ECG for risk stratification in suspected or known CAD in the era of advanced imaging technologies. JACC Cardiovasc Imaging 2015;8:1309-21.

4. Montalescot G, Sechtem U, Achenbach S, et al. 2013 ESC guidelines on the management of stable coronary artery disease. Eur Heart J 2013;34:2949-3003.

5. Knuuti J, Ballo H, Juarez-Orozco LE, et al. The performance of noninvasive tests to rule-in and rule-out significant coronary artery stenosis in patients with stable angina: a meta-analysis focused on post-test disease probability. Eur Heart J 2018;39: 3322-30.

6. Kligfield P, Lauer MS. Exercise electrocardiogram testing: beyond the ST segment. Circulation 2006;114:2070-82.

7. Dunbar CC, Saul BI, Kassotis JT. Exercise testing in the presence of complete heart block. Med Sci Sports Exerc 2007;39:1452-6.

8. Martí-Almor J, Cladellas M, Bruguera J. Atrioventricular block induced by exercise is not always infrahisian. Rev Esp Cardiol 2005;58:1247-8.

9. Soar J, Nolan JP, Böttiger BW, et al. European Resuscitation Council Guidelines for Resuscitation 2015: Section 3. Adult advanced life support. Resuscitation 2015;95:100-47.

10. Hemann BA, Jezior MR, Atwood JE. Exercise-induced atrioventricular block: a report of 2 cases and review of the literature. J Cardiopulm Rehabil 2006;26:314-8.
11. Yandrapalli S, Harikrishnan P, Ojo A, et al. Exercise induced complete atrioventricular block: Utility of exercise stress test. J Electrocardiol 2018;51:153-155.

12. Egred M, Jafary F, Rodrigues E. Exercise induced atrio-ventricular (AV) block: important but uncommon phenomenon. Int J Cardiol 2004;97:559-60.

13. Shetty RK, Agarwal S, Ganiga Sanjeeva NC, Rao MS. Trifascicular block progressing to complete AV block on exercise: a rare presentation demonstrating the usefulness of exercise testing. BMJ Case Rep 2015;2015.

14. Sarullo FM, Accardo S, D'Antoni P, et al. Exercise induced atrioventricular (AV) block during nuclear perfusion stress testing: a case report. Monaldi Arch Chest Dis 2008;70: 29-33.

15. Ho WJ, Chu PH, Cheng NJ, et al. Exercise-induced myocardial ischaemia complicated by paroxysmal complete atrioventricular block. Int J Clin Pract Suppl 2005;59:19-22.

16. Simantirakis E, Marketou M, Kehagias E, et al. Symptomatic exercise-induced complete atrioventricular block due to severe superior vena cava stenosis. Int J Cardiol. 2016;215:167-8.

17. Rumoroso JR, Montes Orbe PM, Cembellín JC, et al. [Exerciseinduced atrioventricular block. Significance of the ischemic component. Report of 4 new cases].[Article in Spanish]. Rev Esp Cardiol 1997;50:278-82.

18. Vilela EM, Bastos JC, Rodrigues RP, Nunes JP. High-sensitivity troponin after running - a systematic review. Neth J Med 2014;72:5-9.

19. Fontes-Carvalho R, Gonçalves-Teixeira P, Vilela EM. The effect of exercise training in systolic and diastolic function. In: Watson RR, Zibadi S, eds. Lifestyle in heart health and disease. London: Academic Press; 2018; pp. 153-62.

20. Vilela EM, Bettencourt-Silva R, Nunes JP, Ribeiro VG. BNP and NT-proBNP elevation after running - a systematic review. Acta Cardiol 2015;70:501-9. 